\title{
WIDE CLASS OF NEW FRACTAL LIGHT SOURCES
}

\author{
Graham S. McDonald ${ }^{1}$, James M. Christian ${ }^{1}$, Jungang G. Huang ${ }^{2}$ \\ ${ }^{1}$ Joule Physics Laboratory, School of Computing, Science and Engineering, \\ Institute for Materials Research, University of Salford, Salford M5 4WT, UK \\ Phone: 44-161-2955079, Fax: 44-161-2955575, e-mail: g.s.mcdonald@salford.ac.uk \\ ${ }^{2}$ Advanced Technology Institute, School of Electronics and Physical Sciences, \\ University of Surrey, Guildford, Surrey GU2 $7 X H$, UK \\ Phone: 44-148-3686086,e-mail:j.huang@surrey.ac.uk
}

\begin{abstract}
Pattern emergence in Nature's complex systems is mostly attributed to a classic Turing instability. There, a single length-scale becomes dominant and this defines a simple emergent structure (for example, a striped or hexagonal pattern). We have investigated whether a multi-Turing instability may result in another universal type of pattern: fractals. Fractals possess proportional levels of detail across decades of length-scale, and are thus inherently scale-less. Here, we make the first predictions of spontaneous spatial fractal patterns in nonlinear ring cavities. This will include the first reported spatial optical fractals arising from purely-absorptive nonlinearity. Analyses reveal characteristic (multi-Turing) spectral features for both dispersive and absorptive cavities. Simulations verify and quantify the fractal properties of the spontaneously-patterned light. Our findings greatly widen the scope for potential realization and exploitation of fractal light sources. In Nature-inspired device and system architectures, such sources are likely to play a pivotal role in developments.
\end{abstract}

Keywords: unstable cavity laser, pattern formation, Turing instability, fractals, ring cavity.

\section{UNSTABLE-CAVITY SYSTEMS: LINEAR FRACTALS}

The unstable-cavity laser is an archetypal system for generating optical fractal patterns [1]. Fractality of the transverse eigenmode patterns arises from a subtle interplay between the eigenmode condition, round-trip image magnification, and layers of successive diffraction patterns [see Fig. 1(a)]. These fractals are linear in the sense that they comprise a linear superposition of linearly-diffracted patterns. Each contributing pattern has an increased characteristic length-scale relative to the previous one. The term "kaleidoscope laser" [2] was introduced to describe systems in which the aperturing element (e.g. the small convex mirror) has polygonal symmetry. In our most recent contributions to this area [3], we have developed methods for calculating arbitrary-order eigenmode profiles of kaleidoscope lasers to unprecedented accuracy [see Fig. 1(b)]. Previous calculations had been limited to either: low Fresnel number cavities (for which mode fractality is over a very limited range of scales); or cavities with asymptotically-high Fresnel numbers. These new developments required novel compact formulations of Fresnel diffraction for arbitrary-shape apertures, and these methods may yield

(a)

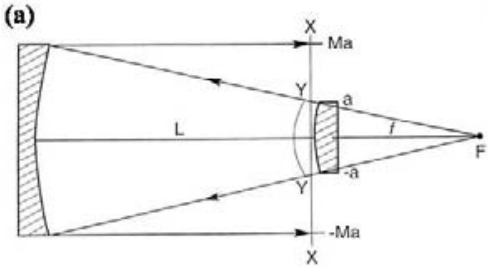

(b)

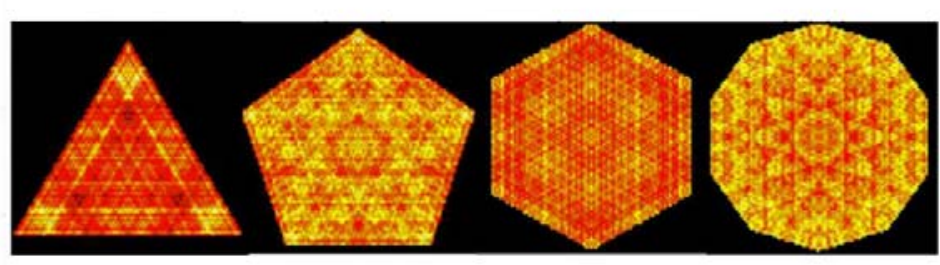

Fig. 1 - (a) geometric rays in an unstable-cavity laser with round-trip magnification $M$. The small mirror acts as an aperture, which leads to small-scale diffraction patterns. (b) transverse intensity patterns of the lowest-order fractal laser modes in resonators with triangular-, pentagonal-, hexagonal-, and decahedral-shaped small mirrors. 
(a)

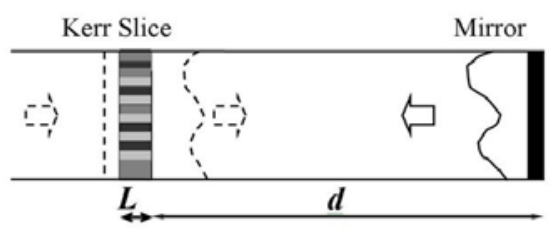

(b)

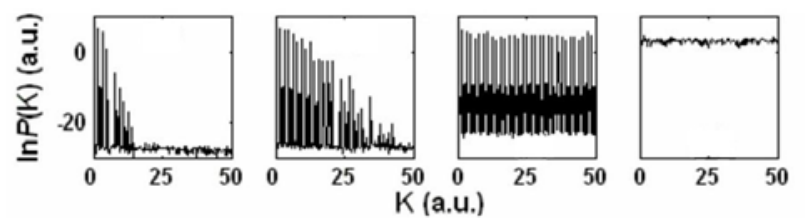

Fig. 2 - (a) counter-propagating light beams in a Kerr slice plus single feedback mirror system. (b) a sequence of frames that illustrates the temporal development of the optical power spectrum ( $K$ is the spatial wavenumber). The 1D spectrum of a simple pattern spontaneously transforms into the spectrum of a (space-filling) fractal.

insight into a range of general phenomena (such as excess quantum noise) - in addition to opening new avenues of exploration regarding the fascinating properties of fractal laser light.

\section{SPONTANEOUS NONLINEAR FRACTALS: (I) SINGLE-FEEDBACK-MIRROR SYSTEMS}

Nonlinear systems are capable of generating spontaneous patterns. The classic mechanism for such a process is a "Turing instability". Here, uniform states of a system those homogeneous in space and stationary in time - can be unstable against arbitrarily-small pattern perturbations [4]. Simple patterns, such as stripes, and hexagonal or square arrays of spots, are well-known to emerge and, subsequently, to characterize the long-term developed states. Importantly, classic Turing instability has been long associated with the emergence of a single dominant length-scale. We identified a wide class of nonlinear optical systems that may be susceptible to multiple Turing instabilities. Furthermore, we proposed that these systems might give rise to spontaneous nonlinear fractals [5]. The nature of such potential multi-scale patterns would clearly be physically distinct from the (purely-linear) fractal eigenmodes of unstable-cavities [1-3], and also distinct from fractal patterns that have been proposed in soliton-supporting systems (where the addition of each new length-scale would require a separate material discontinuity [6]). Instead of these physical origins, our proposed optical fractals would be entirely spontaneous structures, whose emergence and development was driven solely by intrinsic nonlinear dynamics.

Initial investigations of potential spatial fractals in nonlinear spontaneous pattern formation were made in studies of the classic diffusive-relaxing Kerr slice with a single feedback mirror [see Fig. 2(a)]. When some form of spatial filtering is included in the free-space path, this familiar system can generate spontaneous simple optical patterns - such as hexagonal arrays of spots. On removal of this additional filtering, certain parameter regimes were indeed discovered to give rise fractal pattern formation [see Fig. 2(b)]. This proved to be true even when the diffusive medium characteristics suggested that larger-scale Turing instabilities might dominate the long-term outcomes [5]. We were further able to characterize the fractality of the generated light patterns in terms of system parameters - such as the material diffusion length, and the optical pump intensity.

\section{SPONTANEOUS NONLINEAR FRACTALS: (II) RING-CAVITY SYSTEMS}

Our most recent work has examined the possibility of spontaneous nonlinear spatial fractals in ring-cavity systems [see Fig. 3(a)]. Two distinct classes of intra-cavity nonlinear medium - a purely-dispersive Kerr material [see Fig. 3(b)] and a Maxwell-Bloch saturable absorber [see Fig. 3(c)] - have been investigated. We have confirmed that regimes of multiTuring instability can be associated with spontaneous fractal formation for both of these nonlinear ring-cavity systems. 
(a)

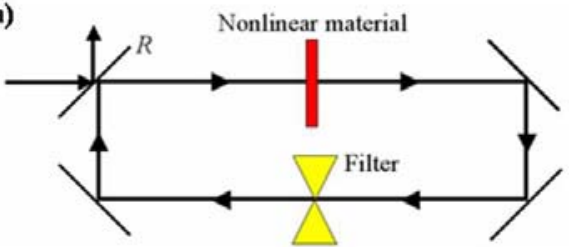

(b)

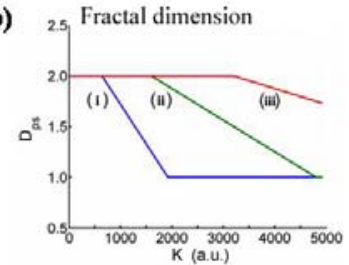

(c)

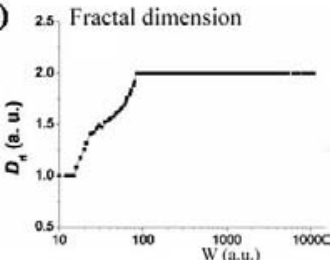

Fig. 3. - (a) a schematic of a wide class of new (ring-cavity) fractal light sources. The filter is introduced only to control the growth of spatial bandwidth. (b) Power-spectrum fractal dimension vs. spatial wavenumber $K$, for optical patterns spontaneously generated when the nonlinear material is diffusive-dispersive (curves are for 3 levels of material diffusion). (c) Optical roughness-length fractal dimension vs. length-scale $W$, when a purely-absorptive nonlinear material is in the ring cavity.

We believe that we have thus confirmed that this multi-Turing instability characteristic is a universal signature of fractal pattern formation in nonlinear wave-based systems. In other words, we have confirmed that prediction of the capacity for spontaneous fractal generation is independent of the particular form of the nonlinearity considered (e.g. dispersive versus absorptive nonlinear systems) and that it is independent of the details of the system configuration (e.g. bi-directional versus uni-directional wave interactions). Consequently, there may be an even wider class of contexts in nonlinear science (i.e. optical and non-optical) where this fractal-generating signature applies. Moreover, to our knowledge, our results include the first predictions of fractal pattern formation in purely absorptive systems [see Fig. 3(c)].

\section{CONCLUSIONS}

Understanding the generation of fractal patterns in nonlinear systems is of fundamental interest. In the general field of spontaneous pattern formation, consideration of the additional roles played by coherence, multi-stability, boundary conditions, and symmetries - along with pattern localization, defects, competition and fronts - presents a wealth of exciting new avenues of investigation. In photon science, our results greatly widen the scope for potential realization and exploitation of fractal light sources. Such sources may play a pivotal role in future nature-inspired devices and system architectures. For example, the huge spatial bandwidth associated with such sources might be harnessed within different technological contexts - such as in probing, ablation, scanning, and information processing applications.

\section{REFERENCES}

1. G. P. Karman, G. S. McDonald, G. H. C. New, and J. P. Woerdman, "Fractal modes in unstable resonators," Nature, vol. 402, p. 138, 1999.

2. G. S. McDonald, G. P. Karman, G. H. C. New, and J. P. Woerdman, "Kaleidoscope Laser," J. Opt. Soc. Am. B, vol. 17, p. 5245, 2000.

3. J. G. Huang, J. M. Christian, and G. S. McDonald, "Fresnel diffraction and fractal patterns from polygonal apertures," J. Opt. Soc. Am. B, vol. 23, p. 2768, 2006.

4. A. M. Turing, "The chemical basis of morphogenesis," Phil. Trans. Roy. Soc. London B, vol. 237, p. $37,1952$.

5. J. G. Huang and G. S. McDonald, "Spontaneous optical fractal pattern formation," Phys. Rev. Lett., vol. 94, Art. No. 174101, 2005.

6. S. Sears, M. Soljacic, M. Segev, D. Krylov, and K. Bergman, "Cantor Set Fractals from Solitons," Phys. Rev. Lett., vol. 84, p. 1902, 2000. 